\title{
Evaluating a dementia learning community: exploratory study and research implications \\ (1) CrossMark
}

Rod Sheaff ${ }^{*}$ (D), lan Sherriff ${ }^{2}$ and Catherine Hagan Hennessy ${ }^{3}$

\begin{abstract}
Background: Access times for, the costs and overload of hospital services are an increasingly salient issue for healthcare managers in many countries. Rising demand for hospital care has been attributed partly to unplanned admissions for older people, and among these partly to the increasing prevalence of dementia. The paper makes a preliminary evaluation of the logic model of a Dementia Learning Community (DLC) intended to reduce unplanned hospital admissions from care homes of people with dementia. A dementia champion in each DLC care home trained other staff in dementia awareness and change management with the aims of changing work routines, improving quality of life, and reducing demands on external services.

Methods: Controlled mixed methods realistic evaluation comparing 13 intervention homes with 10 controls in England during 2013-15. Each link in the assumed logic model was tested to find whether that link appeared to exist in the DLC sites, and if so whether its effects appeared greater there than in control sites, in terms of selected indicators of quality of life (DCM Well/III-Being, QUALID, end-of-life planning); and impacts on ambulance call-outs and hospital admissions.

Results: The training was implemented as planned, and triggered cycles of Plan-Do-Study-Act activity in all the intervention care homes. Residents' well-being scores, measured by dementia care mapping, improved markedly in half of the intervention homes but not in the other half, where indeed some scores deteriorated markedly. Most other care quality indicators studied did not significantly improve during the study period. Neither did ambulance call-out or emergency hospital admission rates.

Conclusions: PDSA cycles appeared to be the more 'active ingredient' in this intervention. The reasons why they impacted on well-being in half of the intervention sites, and not the others, require further research. A larger, longer study would be necessary to measure definitively any impacts on unplanned hospital admissions. Our evidence suggested revising the DLC logic model to include care planning and staff familiarisation with residents' personal histories and needs as steps towards improving residents' quality of life.
\end{abstract}

Keywords: Dementia, Dementia Learning Community, Logic model, Plan-Do-Study-Act, Unplanned admissions, Residential care, England

\section{Background \\ Policy}

After the 2008 financial crash, austerity conditions and policies have made hospital costs and overload an increasingly salient health policy issue in many countries. In the UK, reducing hospital bed use became a pillar of fiscal control, since $67.5 \%$ of NHS costs arise from hospital care [1]. UK policy makers attributed the rising

\footnotetext{
* Correspondence: r.sheaff@plymouth.ac.uk

${ }^{1}$ School of Government, Plymouth University, Drake Circus, Plymouth PL4 8AA, UK

Full list of author information is available at the end of the article
}

demand for hospital care above all to unplanned admissions for older people. It has been known since the 1960 s that older people, especially frail older people, used a disproportionately high share of NHS beds [2-4] partly because hospitals were often providing essentially residential care in the absence of alternative provision [2]. More recently, UK policy makers have attributed part of this bed use to the increasing prevalence of dementia, which became a prominent health policy issue and remit of one of the Prime Minister's three special working groups on healthcare. 
For people who require care and cannot care for themselves (or be cared for) at home, even clinically 'unnecessary' hospital admissions can only be avoided if nonhospital ('community based') residential care is available for them. The NHS has therefore became increasingly reliant on intermediate care, nursing and care homes as means for containing demand for in-patient care. Meanwhile local government budgets (the largest source of finance for residential care in the UK) have been cut, so that families often have to contribute to care home costs. First admission to residential care is tending to occur later in the progression of dementia making care homes' casemix more dependent and complex. In 2012 some 320,000 ( $80 \%$ of) people residing in UK care homes had dementia or severe memory problems. Only $41 \%$ of family members thought that these settings gave the person with dementia a good quality of life [5]. Furthermore the workforce in UK care homes is very casualised, with high rates of staff turnover. There are no official requirements for staff working in care homes to have any prior training [6-8]. $86 \%$ of care home staff felt that providing care to people with dementia was challenging; almost half the staff respondents reported that they want more training in this area [5]. It has become increasingly necessary to develop care homes' capacity to anticipate and prevent health deterioration which might otherwise require unplanned hospital admission. Other health systems face similar issues.

\section{Dementia learning community logic model}

Although the UK currently has no regulatory requirement for residential care staff to be trained in dementia care, its government has recently set targets for such training $[6,7]$. At the time of this study, the immediate training need was therefore for some training at all, starting at the most basic level. Accordingly, quality improvement programmes including specific strategies for dementia are developing. One influential example was the county-wide, multiagency Dementia Link Worker (DLW) scheme in Gloucestershire, which reportedly reduced referrals to NHS services [9]. One component of the scheme - a training programme ('Dementia Leadership Award') aimed at care home owners, managers and clinical leads - was reported to produce the culture change necessary for understanding and supporting DLWs' role [10]. This finding reflects the growing importance of leadership as a concept in dementia education when resource shortages constrain the extent of staff training available and those trained must act as change agents to realise the benefits of this education in practice [11]. Cultivating leadership, increasing staff knowledge and support, and networking informational resources are all elements of interventions involving 'dementia champions', a role which has been employed in various forms in both acute and residential care settings in the UK and elsewhere [12-16]. Reports on the use of dementia champions in residential care have described barriers and facilitators to the uptake of activities arising from this role [14, 15]. However studies which quantify the outcomes of care home initiatives using dementia champions are until now lacking.

Staff in an NHS Trust in England wished to meet similar training needs in their own locality, so as to stimulate organisational changes in care homes, thereby improving care home services and reducing demands on NHS services. To devise a way of doing so they first drew upon published studies (then scarce), press reports (professional periodicals, BBC, on-line) and site visits to other similar projects. They then undertook an initial qualitative assessment in two care homes in their own locality (which were among those that subsequently piloted the DLC intervention described below). This assessment characterised the homes' resident population with dementia; staff qualifications (NVQ/QCF); the facilities' previous dementia training work, its delivery mode and location; care home managers' views of the principal issues in dementia care provision; and their ideas for conducting the training and supporting staff participation during an intervention to address these issues. The NHS staff combined contents from these different sources into an 8-hour multi-module training programme which was developed to include group sessions carried out by the project's dementia learning facilitators (NHS dementia nurse specialists) covering the nature of dementia; principles of communicating with a person with dementia; influence of the environment upon dementia care; person-centred care; care planning and end-of-life care; the Mental Capacity Act and its implications for dementia care; dealing with challenging behaviour; and creating and managing organisational change using Appreciative Inquiry (a method that focuses on exploiting an organisation's existing strengths [17]).

The training was to be the first step in a more complex intervention, the 'Dementia Learning Community' (DLC) model. Like any such intervention the DLC model embodied a specific 'programme theory'. Alongside normative assumptions about what policy outcomes are desirable it contained a 'logic model'. In practice often partly explicit and partly implicit, such a 'theoryin-use' [18] logic model specifies the intervention's activities and resources, and what outputs and outcomes those who make the intervention assume will result [19]. The DLC logic model was as follows:

1. Dementia learning facilitators identify in each care home a 'dementia champion' who is then trained, with other staff, in dementia awareness and how to conduct 'Plan-Do-Study-Act' (PSDA) cycles to improve quality of life [20-22]. Subsequently the learning facilitators regularly visit each dementia champion and use networking activities (teleconferences; web-based forum; monthly team 
awards; newsletter; annual conference) to help the dementia champions to develop leadership skills and confidence; clarify staff roles in the homes; promote best practice in person-centred care; improve care planning; and enhance the care environment.

2. These activities would

(a) increase staff job-satisfaction and self-development; and impart knowledge, attitudes, behaviours and skills associated with best practice in dementia care.

(b) initiate and sustain PDSA cycles including care audits and quality improvement and spread activities in each care home.

3. Together the impacts at step 2 above would result in everyday care home work routines increasing residents' well-being.

4. Longer term, the improved work routines would raise the residents' health-related quality of life. Better planning of care and better monitoring of residents' condition would lead to earlier observation of emerging health-related problems, and therefore to remedial action (i.e. secondary prevention) to prevent these problems developing.

5. Raising their health-related quality of life would reduce residents' need for ambulance call-outs and unplanned (emergency) hospital admissions.

The facilitators' follow-up sessions with the dementia champions converted a training programme into a PDSA intervention and were the rationale calling it a 'learning community'. The DLC model was therefore novel in linking attempts at culture change in care homes strongly to the development of PDSA activities. Such methods for improving service quality are extensively researched in hospitals but not care homes, so findings from the initial evaluative research which this paper reports may have wider interest and application.

The above logic model is what we tested in this evaluation. Figure 1 shows the main causal links (labelled 'A'-'F') which the DLC logic model assumed.

Links A, B and D apply only to DLC sites. The more generic links $\mathrm{C}, \mathrm{E}$ and $\mathrm{F}$ would be found in both there and elsewhere, but with stronger effects in DLC sites. In practice each link is likely to be confounded and one would expect the effect of the initial training to become attenuated at each step. Training alone has more impact on care workers' knowledge of dementia than on their coping styles [23] hence may not be enough to raise quality of life for people with dementia [24]. Indeed training in safeguarding vulnerable adults and longer time working in the sector may reduce trainees' confidence, possibly because better-informed staff become less complacent that residents' needs are being properly safeguarded, although the greater the apparent deficiencies in care, the more confident trained staff become about reporting them [25]. Dementia Care Mapping (DCM: see below) may in itself help reduce staff burnout [26] so that improved service quality feeds back into improved staff morale. Person-centred care appears to reduce agitation [27] and use of antipsychotic drugs [28] in people with dementia, and to improve staff recognition of cognitive impairment in residents [29]. Research on the effects of quality improvement and spread methods, particularly PDSA cycles [30] mostly concern acute hospitals. Studies set in services for older people

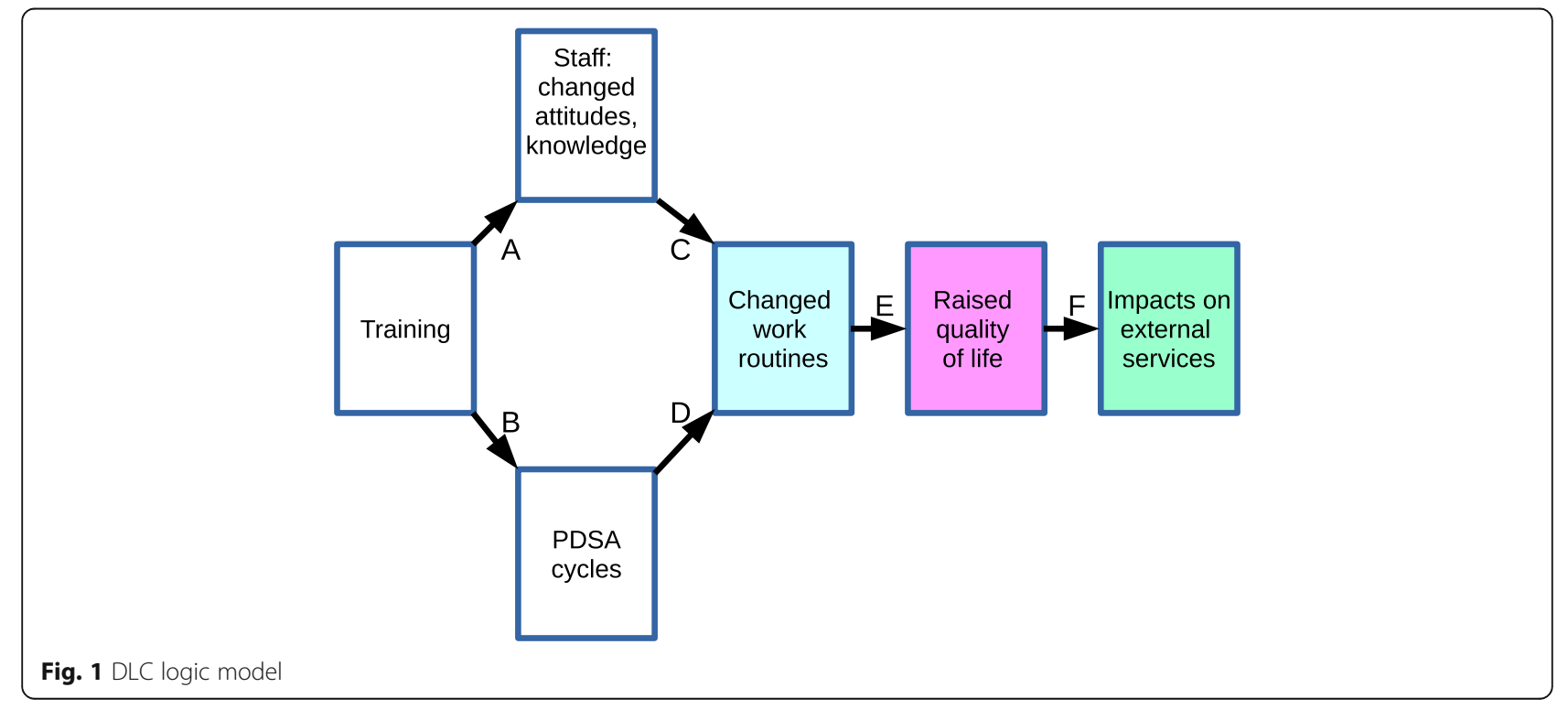


with cognitive impairment are scarce but also tend to report positive outcomes [21,31,32]. However residential care work routines are not the only influence on residents' quality of life, and the latter is not the only determinant of unplanned hospital admissions [33]. Similar qualifications apply to studies of the effects of networking in sustaining changes in work routines (e.g. showing that inter-nursing home collaboration improves uptake of pain-management methods [34]) or in improving mental health care as rated by patients and carers [35].

\section{Research questions}

In the absence of studies evaluating the DLC logic model, we aimed to make a preliminary exploratory attempt to evaluate it empirically. It was exploratory in that we aimed not only to assess how far the model had been implemented and its effects, but in doing so also to elicit, test and refine the underlying 'theory-in-use' logic model of how a DLC works. In that way we could assess whether, or with what modifications, the DLC model might be reproducible elsewhere. By exploring the effects and their magnitudes we also could lay the basis, if the findings warranted it, for a more definitive and detailed evaluation that would supplement the evidence base for care for people with dementia. We therefore addressed the following research questions. Each corresponds to a stage in the logic model.

In the care homes that participated:

RQ 1. Were the initial training and facilitation implemented as specified?

RQ 2. What was the impact on staff job satisfaction, knowledge and attitudes about dementia and confidence in providing care to persons with dementia?

RQ 3. What PDSA activities resulted?

RQ 4. What ensuing changes in work routines and therefore resident well-being could be attributed to the impacts on staff job satisfaction, knowledge, attitudes about dementia and confidence and/or to the PDSA activities?

RQ 5. How did measured quality of life change for residents?

RQ 6. What were the impacts on ambulance call-outs and emergency hospital admissions?

\section{Methods}

\section{Design}

Since any policy or organisational intervention tacitly applies a logic model [36], a realist approach is to make that logic model explicit and expose it to evidential testing, so that it can be revised as necessary and thereby strengthened. Because we aimed to evaluate the logic model on which its inventors had implicitly based the DCL, realistic evaluation [26] was the study design of choice, not least because that design focuses attention on understanding the context in which an intervention is carried out and how that context (also) influences the observed outcomes [27]; in the present case, determining how the DLC intervention worked in care environments typified by a low-paid, casualised work force with high turnover [8]. We therefore adopted a multi-method realist evaluation [37] research design comprising:

1. An implementation component investigating the logic model's implementation structures [38], what factors facilitated or impeded implementation, or affected its outcomes [39], from the initial training through to the follow-up work to support any ongoing PDSA cycles. This component addressed research questions 1, 2 (partly), 3 and 4 (partly).

2. An impact component testing, link by link, whether each link in the logic model appeared to be present, addressing research questions 2 (partly), 4 (partly), 5 and 6.

A necessary preliminary to both was to elicit the logic model which those who designed and implemented the DLC were using. We did that through discussions, interviews and e-mail correspondence with them, by context-analysing project documentation and websites, and content-analysing research publications that the project implementers said they had used. Some of these materials are cited, and the resulting logic model reported, above.

\section{Setting and sampling}

Both components used the same settings, but the implementation component applied only to the DLC homes in the sample. All 23 study homes were privately owned, representing about a quarter of the care homes in one English local authority. They volunteered to participate in the understanding that those selected as controls could also become DLC sites once the study finished. We randomly allocated homes to intervention (DLC) and control groups by file sampling the list of volunteer care homes (Table 1). Achieving approximately equal numbers of residents in each arm required 23 care homes ( $=10$ controls +13 DLC).

At the outset we compared residents' baseline DEMQOL, QUALID and DCM scores (see below) between the DLC and control sites to check whether the former already appeared to be giving higher-quality care and/or to have residents in better health (in terms of dementia), but there were no significant differences at that stage. 
Table 1 Control and DLC sites

\begin{tabular}{|c|c|c|}
\hline & Control & DLC \\
\hline $\mathrm{N}$ (homes) & 10 & 13 \\
\hline Ownership & $\begin{array}{l}4 \text { corporate; } 6 \\
\text { owner-managed }\end{array}$ & $\begin{array}{l}10 \text { corporate; } 1 \\
\text { owner-managed; } 1 \\
\text { religious; } 1 \text { charitable. }\end{array}$ \\
\hline Residents & $\begin{array}{l}330 \\
\text { (range 20-51) }\end{array}$ & 288 (range 14-46) \\
\hline Staff & $\begin{array}{l}245 \\
\text { (range 17-50) [*] }\end{array}$ & 298 (range 11-53) \\
\hline Mean staff wte per bed & 0.82 (range $0.61-1.03$ ) & 1.02 (range $(0.59-1.58$ ) \\
\hline
\end{tabular}

[*] For 9 homes. No data from one owner-managed home

\section{Implementation component}

To examine whether, and if so how, DLC training had created links A, B and D we used qualitative methods, taking an ethnographic approach $[40,41]$ to discover how the dementia champions in each DLC home were recruited and trained, any PDSA activities, their practical consequences, what was being done to sustain them, and any difficulties or resistance. Training session participants completed review questionnaires, from which we collated their responses about what they had learned and what they intended to do as a result. We interviewed four of the dementia champions and analysed all the network facilitators' contemporary field-notes of their four-weekly meetings with each dementia champion. We analysed these data inductively and thematically [42], seeking patterns across the DLC homes in how the logic model links were, or were not, established in practice.

\section{Impacts component Measures}

We treated each link as having an antecedent (as the case may be, initial DLC training or the later changes it was intended to trigger) and a consequent (impact). All measures except (1) and (6) below were therefore used twice, as the consequent of one link and the antecedent for the next.

1. DLC Intervention: completion of training sessions (yes/no).

2. PDSA cycle: evidence of PDSA activity (yes/no).

3. Changes in staff attitudes, knowledge of dementia and morale were assessed by changes in scores on the: Dementia Attitudes Scale (DAS) [43]; Approaches to Dementia Questionnaire (ADQ) [44]; and the English version of the Swedish Satisfaction with Nursing Care and Work Assessment Scale (SNCW) [45], in which lower scores indicate greater satisfaction with, or a more positive attitude towards, work). As a proxy for staff morale and for constraints on relational continuity of care, we measured sickness leave rates per member of staff per home for the year before and the year after the start of the DLC intervention.

4. Immediate effects of changed work routines on residents were measured by changes in the Welland Ill-Being (WIB) scores used in Dementia Care Mapping (DCM) [46, 47], interpreting increased scores as evidence of changed work routines. We used a two-hour observation frame which is within the range for previously published evaluations [47] and sufficient for psychometric stability [48]. We regarded DCM as a measure, not as an intervention in its own right for making staff more residentcentred in their work [27].

5. Changes in dementia-specific measures of residents' quality of life: We used the Global Deterioration Scale (GDS) [49] to decide whether residents' quality of life should be measured using DEMQOL [50] (a self-response tool) or, for residents with a GDS score of 4 or above (mild dementia/moderate cognitive impairment) the Quality of Life in LateStage Dementia (QUALID) scale [51]. (DEMQOLProxy, an otherwise suitable measure, was not feasible because few residents had non-staff carers to hand.) We also measured the change in number of residents with records of an end of life discussion, end of life care plan, an advanced care plan and a completed treatment escalation plan (TEP) form.

6. External impacts: changes were measured by the proportionate change in homes' annualised rates, per resident, of:

(a) Ambulance call-outs, as reported in the homes' administrative records.

(b) Hospital admissions: both unplanned and planned, using routine administrative data from the local Clinical Commissioning Group.

Because the care home (site) was the unit of analysis, we aggregated individual-level measures to yield mean scores per home, which also removed any clustering in individual-level scores and compensated for the different sizes of homes.

\section{Sampling}

Before starting fieldwork we estimated from published reports $[52,53]$ that a sample of 384 residents $(192+$ 192) was necessary to give a $90 \%$ chance of detecting a $5 \%$ superiority of outcome in the DLC programme, significant at the 5\% level, using DEMQOLv4 [50] as our quality measure or, using QUALID, 632 residents (316+ $316)$. In the event achieving even the smaller sample size was difficult because of the high drop-out rate due to 
the natural progression of dementia, reducing the eventual pre- and post-intervention dataset to 246 residents. Furthermore, GDS rating showed that only 27 residents in the post-intervention dataset were suitable for the DEMQOL measure of quality of life, leaving QUALID as the default.

\section{Analyses}

We tested each link of the logic model in two steps.

First, if a link were present, changes in its antecedent(s) would be associated with changes in its consequent(s). Since DLC training was their antecedent, links A, B and D would be found only in the DLC sites, so only data from those sites could be used to test whether those links existed. The remaining links were generic and should, the logic model implied, exist in all sites. So to bring more data to bear, we tested for those associations across all the study sites.

Second, each link would produce a bigger change in its consequent (staff characteristics, work routines, quality of life or the external impacts, as the case might be) in the DLC sites than controls. For staff characteristics, work routines and quality of life we had only one datapoint available before and one after the DLC intervention. We therefore made those comparisons by testing cross-sectionally for any differences in the proportionate change (i.e. the mean post-intervention score divided by the mean pre-intervention score per home) in these measures between DLC and non-DLC sites. For the external impacts, routine administrative data allowed us to make difference-in-differences analyses. Because sites' mean duration of participation in the DLC was 12.3 months (range 11 to 14 months) we took the preceding 12 months (October 2013-September 2014) as the matched control period. For all statistical tests (Wilcoxon signed rank, linear regression) we used version 3.3.1 of the $\mathrm{R}$ software with a declared significance level of $p \leq 0.05$. (Relative and absolute risks or risk reductions are not relevant to these tests. Confidence intervals are not relevant to Wilcoxon tests.)

\section{Ethics}

All these privately-owned homes fell outside the remit of the NHS and local authority research ethics approval systems. We therefore obtained ethical approval from Plymouth University Research Ethics Committee on 21st February 2014 (Reference PU13/14-216), as a condition of which informants and care homes are pseudonymised below. Informed consent was obtained from all participating care home staff. Consent procedures for residents accommodated their capacity to provide permission to participate [54]: for those with mild to moderate dementia, their willingness to take part in the research was confirmed on an ongoing basis, while for those with severe dementia, consent was sought from a family member or other available proxy with designated authority to provide consent. We obtained individual consent (including consent to audio-record interviews) from individual care home managers and staff members.

\section{Results}

After reporting response rates we present our results for each link in the logic model. For brevity we present only overall scores except where necessary to interpret or disambiguate the results.

DLC trainees' response rate was $100 \%$. For the staff survey (all sites) it was 202/603 (33\%). Staff sickness and turnover data for were available for $18 / 23$ sites (78\%). Pre- and post-intervention Dementia Care Mapping (DCM) data were obtainable for $15 / 23$ sites (65\%), of which 5 were controls and 10 DLC sites. QUALID data were obtained for 246 residents, i.e. $38 \%$ of the study homes' total population. Data on Treatment Escalation Plans were obtained for 201 residents (31\%). Ambulance data for 'See \& Treat' and 'See \& Convey', but not for 'Hear \& Treat', call-outs were available for $21 / 23$ sites (91\%). 62 (i.e. 19\%) of the initial 332 residents in the study had no formal diagnosis of dementia at the start of the evaluation, but 47 of those 62 had a GDS score of 4 or above, indicating 'deficits ... clearly manifest in a detailed clinical interview ... subjects ... who enter this fourth stage almost invariably manifest subsequent deterioration characteristic of dementia of the AD type [55]. Two DLC sites dropped out, one because a local authority had concerns about quality of life there and ceased referrals to it. The other changed ownership. Testing for the presence or strength of a given link is only possible, however, for the sites which supplied data about both its antecedent and its consequent, so the amount of usable data for each link was less, as reported below.

\section{Link a: Training and impacts on staff}

The same training took place in all DLC sites, and they all implemented it as planned. Table 2 shows the main training outcomes that participants reported.

Dementia champions were recruited by asking the care home managers to identify volunteers for the role, which all except two managers did. In default these two managers took on the dementia champion role themselves. As their intentions for working differently in future, trainees mentioned listening to and observing residents more (staff from sites A,F,J,M,U,W); learning more about the residents as individuals from documented information, other staff and residents themselves (sites A,B,F,I,J,R,U); interacting more with residents and spending more time with them (sites A,B,G,J,I,M,Q,R,S,W); helping fellowworkers work more as a group and communicating more 
Table 2 Self-reported training outcomes

\begin{tabular}{ll}
\hline Better prepared for dealing with aggressive resident behaviour & 13 \\
Confidence building & 5 \\
Learn about end of life care & 1 \\
Communicate better & 12 \\
Understanding dementia [named specifically] & 108 \\
'Awareness', 'knowledge', 'learning' [topic unspecified] & 102 \\
No response & 40 \\
Total & 282 \\
\hline
\end{tabular}

with them (sites B,F,G,J,I,Q,W) e.g. by making better use of the home's communication book. A few mentioned giving more 'person centred' care (sites G,M,S) or being more compassionate and tolerant (sites B,G). One, more modestly, intended to 'stop annoying the residents' (site S). Only one trainee asserted that there was 'nothing' he/she wanted to do differently.

As the logic model predicted, DAS and ADQ total scores rose in the DLC sites and the SNCW total scores fell. However the training mostly did not appear to make the staff characteristics change further in the predicted direction in the DLC than in the control sites. Mean DAS total score rose by 2.18 in the DLC group but by 4.39 in the control group, over a scale 120 points long. The difference was not statistically significant (Wilcoxon rank sum $\mathrm{W}=57, p=0.86)$. Neither was the difference for mean ADQ total score, which fell by 0.76 in the intervention group and rose 0.5 in the DLC sites, on a 76 -point-long scale $(\mathrm{W}=41, p=0.42)$. Mean SNCW total score fell 7.53 points in the DLC sites but rose 0.56 points in the control sites, on a scale 128 points long, a difference which was statistically significant $(\mathrm{W}=65, p=$ 0.045 ), because of decreases (separately not quite statistically significant) in the Quality (W=62.5, $p=0.07)$ and Workload (W $=63, p=0.06)$ components of SNCW. The Cooperation (W $=45.5, p=0.47$ ), Development (W $=58, p=0.14)$ and Patient Knowledge $(\mathrm{W}=60.5, p=0.09)$ components of SNCW showed no significant difference. Mean sick days per staff member per year significantly decreased by 1.35 in the DLC sites but increased by 0.42 in the control sites $(\mathrm{W}=54, p=0.05)$. We found no significant difference in staff turnover.

\section{Link B: Training and PDSA cycles}

The initial training did trigger cycles of PDSA activity in all homes that participated. Partly the dementia champions expressed their aims for the PDSA cycles in general, aspirational terms, most often 'To show us the right path regarding the dementia experience' (site J; and, differently worded, sites F, I,Q,R,S,U,W). 'Person centred ('individualised', 'personalised') care' was mentioned in sites G,I,J,Q,R,S and W, and care home culture by the dementia champions for sites A, B and M. However, the dementia champions also stated more concrete, practical aims for their PDSA activity. In descending order of frequency, these aims were: to find out more about the home's residents in order to inform staff interactions with them (sites G,I,M,S,U,W) or, more concretely, by producing one-page resident profiles or 'This is me' notes (sites A,B,F,I,Q,R). A second set of planned activities concerned environmental enhancement for residents, including reviewing care routines and making them more person-centred. These activities included the use of memory boxes (site B), residents helping themselves to vegetables at mealtimes (site $\mathrm{S}$ ); having books, magazines, newspapers, games (cards, dominoes) for all residents to use when they wanted (site U), enabling residents to have personal items to hand (site U) or having their room set up as they liked (site U), and having assisted baths first thing in morning (site G). Staff received further training in dementia awareness at sites J,M,R and W.

\section{Link C staff characteristics and immediate effects of work routines}

Initially the study sites had generally low overall Welland Ill-Being (WIB) scores. Only six sites scored $\geq 2.5$, and only three of them $(B, U, V)$ met the DCM standards across all categories. At the end of the scheme (approximately one year), five DLC homes showed marked changes in their WIB scores but another five did not (Table 3).

In sites $A, B, I, M$ and $S$ the changes in WIB scores were within the same range as those for the control sites, i.e. they either increased by less than $30 \%$ or fell. Indeed DLC sites A and B showed larger falls in WIB score than any of the control sites. In aggregate there was no significant difference between the Low-WIB DLC sites (A,B,I,M and S) and the control sites. A second group of sites (F,R,U,W and Y) showed another pattern. Their WIB scores rose by between $70 \%$ and $742 \%$, a statistically significant mean increase of 1.8 points $(\mathrm{W}<0.1$, $p<0.01)$. For short we label the two groups 'High-WIB' and 'Low-WIB' sites. It therefore appeared that either or both of links $\mathrm{C}$ and $\mathrm{D}$ were present in five 'High-WIB' sites, and stronger there than in the controls.

To test whether link $C$ was present, we tested for association between the measures of staff characteristics in the DLC sites, and in particular for the High-WIB sites taken alone, and the respective WIB Scores. Taking all the DLC sites together, we found no association between the proportionate changes in DAS total score and WIB score $(\mathrm{W}=52, p=0.86)$ or ADQ total score and WIB score $(\mathrm{W}=66, p=0.47)$. The proportionate change in WIB score was however associated with proportionate 
Table 3 WIB score changes

\begin{tabular}{lllll}
\hline Site & Status & PRE Mean WIB & POST Mean WIB & Change \\
\hline D & C & 1.10 & 0.90 & -0.2 \\
H & C & 0.73 & 0.7 & -0.03 \\
O & C & 0.95 & 0.94 & -0.01 \\
K & C & 1.02 & 1.2 & 0.18 \\
V & C & 0.66 & 0.85 & 0.19 \\
A & DLC & 0.89 & 0.00 & -0.89 \\
B & DLC & 1.80 & 1.25 & -0.65 \\
I & DLC & 0.96 & 0.87 & -0.09 \\
S & DLC & 0.49 & 0.54 & 0.05 \\
M & DLC & 0.88 & 1.14 & 0.26 \\
R & DLC & 1.15 & 1.95 & 0.8 \\
F & DLC & 1.34 & 3.21 & 1.87 \\
U & DLC & 1.06 & 3.01 & 1.95 \\
Y & DLC & 0.03 & 2.11 & 2.08 \\
W & DLC & 0.31 & 2.61 & 2.3 \\
\hline
\end{tabular}

PRE pre-intervention, POST post-intervention. C Control site, DLC

intervention site

change in SNCW score, and negatively as predicted (SNCW being reverse-scored) ( $\mathrm{W}=97, p<0.01$ ).

Of the presumed antecedents of link C, ADQ and ADS scores (of staff attitudes and knowledge) did not significantly differ between the DLC and the control sites, but SNCW scores did (W $=65, p=0.05$ ), both for all DLC sites and the High-WIB sites taken separately $(\mathrm{W}<0.01, \mathrm{p}<0.01)$.

\section{Link D: PDSA activity and immediate effects of improved work routines}

We found evidence (both interviews and physical artefacts) at the DLC homes that knowledge from the training sessions had been used to initiate post-training PDSA activities directed at improving working practices there. At the High-WIB sites (F,R,U,W,Y) PDSA activities focused on: elaborating residents' care plans using information gathered at the training session and subsequently (site $\mathrm{U}$ ); periodic staff meetings about residents (site F); designating key-workers responsible for particular tasks and/or residents (site R); setting up routine team meetings so as to re-iterate and sustain future PDSA cycles (site Y); and training staff by letting them experience what life as a care home resident is like (e.g. being fed by someone else, wearing incontinence pads etc.) (site W). However PDSA cycles in low-WIB homes initiated many similar activities. Sites A and I identified key-workers responsible for particular tasks and/or residents, Sites I and S initiated routine team meetings to sustain future PDSA cycles, since 'You can learn all the time about dementia' (Dementia champion). Site B did small-scale initial testing to see if new ideas worked.
Homes $\mathrm{M}$ and $\mathrm{S}$ initiated planning morning activities for residents. Two homes for which we did not have beforeand-after WIB data also reported similar post-training PDSA activity. Periodic staff meetings about residents were introduced at sites $\mathrm{G}$ and $\mathrm{Q}$. In addition site $\mathrm{G}$ identified key-workers responsible for particular tasks and/or residents, established routine team meetings so as to sustain future PDSA cycles, undertook small-scale initial testing to see if ideas worked, and introduced planned morning activities for residents.

So far as we are aware, data from PDSA activities were not usually documented. Neither, therefore, were such data re-used over time. PSDA cycles were predictivelybased only in the sense that participants anticipated certain broadly-defined outcomes from the 'Do' phase (e.g. that residents would become happier). Thus PDSA cycles were implemented, but compliance with the PDSA model [22] was patchy. There was no PDSA activity in the non-DLC sites.

What differentiated the high-WIB and low-WIB sites was not whether PDSA cycles followed the initial training nor, mostly, what the contents (foci) of the PDSA cycles were. So perhaps other factors not recognised in the logic model, such as the organisational character of High-WIB homes, were responsible instead. High-WIB homes tended to be larger (a mean of 29 beds versus $25.8)$ and have a higher staffing ratios (1.16 wte staff per resident versus 0.93) but in our data these differences were not statistically significant. High-WIB and LowWIB sites did not differ in terms of the aspirations stated in the post-training questionnaires and follow-up sessions, home location or ownership type. The only resistance to DLC activity was in Low-WIB sites. In one, the manager reported that some staff were suspicious and defensive about the DLC and would not complete residents' 'This is me' documents. That and another LowWIB site had difficulty recruiting dementia champions. The absence of link $C$ leaves only PDSA activity (link D) to explain the increased WIB scores in the five 'HighWIB' DLC sites, but apart from eliminating home location, size, staffing and ownership the data available to us did not reveal what other contextual differences between High-WIB and Low-WIB homes enabled PDSA activities in the former to change working practices sufficiently to raise their WIB scores.

\section{Link E: Effects of improved work routines and quality of life}

At baseline, not one site scored better than the midpoint of the QUALID scale. Taking DLC and control sites together, change in WIB score was associated with proportionate change in total QUALID score $(\mathrm{W}=155$, $p=0.03$ ) and in each QUALID component, although not in the High-WIB homes taken alone. Comparing DLC 
and non-DLC sites, total QUALID scores fell in both intervention and control homes, by a mean of -1.68 and -0.57 respectively for a 44-point scale, but the difference was not statistically significant (W $=39, p=$ 0.23). We found the same pattern for each separate component of QUALID. We also compared only the High-WIB homes with the controls, but again found no significant differences. Considering the high mortality among this population it was striking how few end-oflife care plans there initially were; 42 in the control and DLC homes combined (646 beds). There was no difference between DLC and non-DLC homes in the change in frequency of use of end-of-life care discussions, care plans, or TEP forms.

\section{Link F: Quality of life and external impacts}

Contrary to the logic model, improved QUALID scores were, across all sites, associated with increased (not reduced) ambulance call-outs (by an average of 1.6 callouts per year per home) $(\mathrm{W}=343, p=0.03)$. Comparing all DLC sites with the controls also showed no significant difference in the change in rate of ambulance callouts ( $\mathrm{W}=70, p=0.06)$. In respect of ambulance callouts, link $\mathrm{F}$ was not present.

Across all the sites, changes in QUALID total scores were not associated with changes in the rate of all admissions $(\mathrm{W}=373, p=0.08)$, nor with changes in the rates of emergency admissions specifically $(\mathrm{W}=295, p=$ 0.3 . At the start of the study DLC sites already had lower admission rates (both planned and unplanned) than the control sites. Nearly all hospital admissions (374/389, 96\%) from all the homes were as emergencies. During the project the number of admissions per bed per year did fall in the DLC sites (by $20 \%$ for all admissions and $27 \%$ for emergencies) but since admissions also fell in the control sites the difference-in-differences analysis showed that for all admissions the 'treatment' (i.e. DLC) effect was negligible ( $\delta$ approaching zero). For emergency admissions it was in the predicted direction but small $(\delta=-0.3$ emergency admissions per bed per year), and still not statistically significant $(p=0.29)$. For hospital admissions too link $\mathrm{F}$ was absent.

\section{Discussion}

Our methods assumed that no confounding change occurred during the DLC project, and no 'contamination' of the non-DLC sites with DLC work routines, even though DLC and control sites were often nearby and staff turnover (hence transfer between workplaces) was frequent. Also our methods assumed a comparable case-mix during the two years of the study, and between homes, despite high mortality among residents. Having volunteered as DLC sites, one might expect the study homes to be if anything more motivated than other care homes to implement and exploit the DLC model. Comparing the above results with those of an initial pilot analysis of the external impacts six months into the study, it was noticeable how rapidly the $p$-values fell towards significance as the quantity of data increased, raising the question of whether a longer evaluation might yield results more favourable to DLC. Evaluations of other 'culture change' interventions in residential longterm care suggest the importance of allowing interventions enough time to mature or 'bed down' before evaluating them [56]. Our results also suggested that if the observed changes in emergency admissions were indeed due to the DLC, they took over six months to appear.

As reported above, ADQ and ADS scores (of staff attitudes and knowledge) did not significantly differ between the DLC and the control sites, but SNCW scores did. We also found that the location, size, staffing and ownership of High-WIB homes, did not appear to explain why Link D, beween PDSA activity and immediate effects of improved work routines, differed between high-WIB and low-WID sites. These results suggest revising the logic model, and in particular re-interpreting link C.

\section{Conclusion}

\section{Summary results}

We aimed to make a preliminary, exploratory empirical evaluation of the DLC and its underlying logic model. In summary we found equivocal evidence for the presence of links A,B,C,D and E, and none for link F (Table 4).

Our impact and implementation data both suggested that any effects which the DLC training had upon staff characteristics (link C) came through encouraging staff to know more about their residents and improving staff morale ('Development') rather than by changing knowledge or (other) attitudes. This is consistent with other studies demonstrating that the effects on practice of dementia education interventions with residential care staff are best achieved when didactic training is accompanied by experiential learning and support [57]. PDSA activities appeared to be the more 'active ingredient' in the DLC programme, but compliance with the PDSA model was patchy. The contrast between High-WIB and LowWIB DLC sites suggested that care improvement depended on how a home implemented its PDSA activity, raising the contextual question of what the HighWIB homes did differently to the other DLC sites (and the controls), for example in terms of sustaining the cycles long-term and adapting them [22]. Finding staff resistance raises questions about what factors affect fidelity of implementation of PDSA activities in residential care. Our results confirmed that one should expect any effects of the DLC would weaken through successive links of the logic model. 
Table 4 Empirical status of DLC logic model

\begin{tabular}{|c|c|c|c|c|c|}
\hline \multirow[t]{2}{*}{ Link } & \multirow[t]{2}{*}{ Antecedent } & \multirow[t]{2}{*}{ Consequent } & \multirow{2}{*}{$\begin{array}{l}\text { Measure(s) of } \\
\text { consequent }\end{array}$} & \multicolumn{2}{|l|}{ results } \\
\hline & & & & Link found? & Consequent ('effect') stronger in DLC? \\
\hline \multirow[t]{3}{*}{ A } & \multirow[t]{4}{*}{$\begin{array}{l}\text { DLC training } \\
\text { (DLC sites only) }\end{array}$} & \multirow[t]{3}{*}{$\begin{array}{l}\text { Staff } \\
\text { characteristics }\end{array}$} & DAS, ADQ, SNCW & Yes & $\begin{array}{l}\text { No for DAS, ADQ and parts of SNCW. } \\
\text { Yes for SNCW Development and Patient } \\
\text { Knowledge components. }\end{array}$ \\
\hline & & & Sick-leave & Yes & Yes \\
\hline & & & Turnover & No & No \\
\hline B & & PDSA cycles & $\begin{array}{l}\text { Reported/not } \\
\text { reported }\end{array}$ & Yes & Yes \\
\hline C & Staff characteristics & \multirow{2}{*}{$\begin{array}{l}\text { Immediate impact } \\
\text { of work routines }\end{array}$} & \multirow{2}{*}{$\begin{array}{l}\text { DCM WIB } \\
\text { scores }\end{array}$} & Partly: for SNCW but not DAS, ADQ. & No \\
\hline $\mathrm{D}$ & PDSA & & & Yes, but 'High-WIB' homes only & $\begin{array}{l}\text { Yes, but 'High-WIB' } \\
\text { homes only }\end{array}$ \\
\hline \multirow[t]{4}{*}{$\mathrm{E}$} & \multirow{4}{*}{$\begin{array}{l}\text { Immediate impact } \\
\text { of work routines }\end{array}$} & \multirow[t]{4}{*}{ Quality of life } & QUALID & Yes & No \\
\hline & & & $\begin{array}{l}\text { End-of-life care } \\
\text { discussion }\end{array}$ & No & No \\
\hline & & & $\begin{array}{l}\text { End-of-life } \\
\text { care plans }\end{array}$ & No & No \\
\hline & & & $\begin{array}{l}\text { TEP decision } \\
\text { forms }\end{array}$ & No & No \\
\hline \multirow[t]{2}{*}{$\mathrm{F}$} & \multirow[t]{2}{*}{ Quality of Life } & \multirow[t]{2}{*}{ External impacts } & $\begin{array}{l}\text { Ambulance } \\
\text { call-outs }\end{array}$ & No & No \\
\hline & & & Emergency admissions & No & No \\
\hline
\end{tabular}

\section{A revised logic model}

Nevertheless our findings also show the feasibility of implementing the DLC model, what effects can be expected from it over what timescale, and - subject to further research (see below) - which contexts may help produce those effects. Among other things, the training and PDSA activities stimulated not narrowly clinical monitoring so much as staff familiarisation with residents' present state, background, personal interests, preferences and needs; and improved care planning. In turn these activities improved residents' quality of life. The original logic model did not clearly differentiate these sequential events. Above we infered that link $\mathrm{C}$ in the original logic model required reinterpretation. The relevant 'knowledge and attitudes' appeared to be attitudes towards staff development and knowledge about residents (part of what the SNCW records). Since PDSA cycles appeared to be a key 'active ingredient' in the DLC model, knowledge and practical skills in carrying them out should also be considered part of link $\mathrm{C}$. The more immediate and reliable outcome of DLC model lay in using PDSA cycles to improve health-related quality of life for care home residents with dementia. Reduced use of hospital services was a less certain, longer-term outcome. We therefore propose modifying it as fig. 2 shows.

Besides making a preliminary empirical evaluation, this study also contributes to knowledge by suggesting, in light of the above evidence, potential revisions to the DLC logic model to improve the intended outcomes in these and other settings. The revised logic model (figure 2) may be of practical use in guiding further development and applications of the DLC model, thereby contributing to strengthening the quality of the dementia care environment and the contribution of training to doing so.

\section{Research implications}

We found prima facie evidence for parts of the DLC logic model, which we therefore conclude shows sufficient promise to merit fuller evaluation. Our results also expose the challenging case-mix, low quality of care and organisational instability of some care homes in the locality studied. The DLC intervention was developed to be transferrable and sustainable across the large number of care homes served by the NHS Trust. To implement other recognised practice improvement approaches across so many homes would not at present be feasible. Data limitations and the 'on-balance' character of some results mean that the present study should be regarded as initial exploratory research, containing findings (e.g. concerning recruitment, resources, methodologies, acceptability, outcome measures, potential effect sizes and contextual appropriateness [21]) relevant to designing any future RCT or quasi-experimental evaluation and showing how the elements of such a research study could work together. The case-mix which we found suggests either that QUALID is the more relevant quality measure for this care group, or that a much larger study would be needed if DEMQOL were a required outcome 


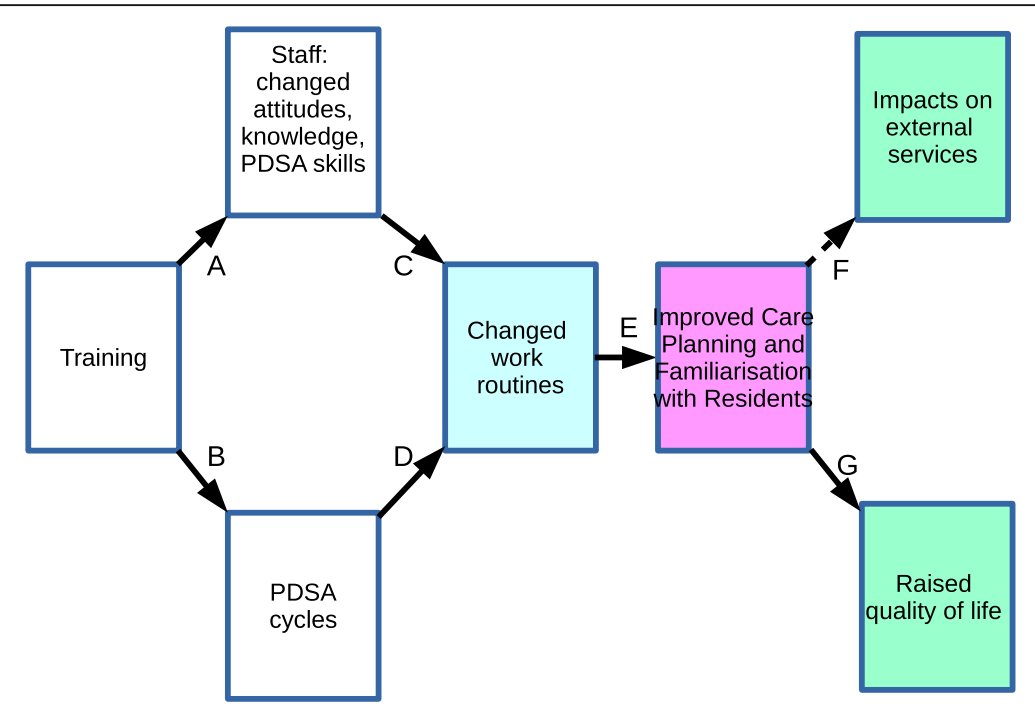

Fig. 2 Revised DLC logic model

measure. A larger study would also be required for obtaining more certain results about DLC impacts on ambulance call-outs, hospital admissions and (not covered here) prescribing rates for anti-psychotics. The drop-out rates reported above also have implications for estimating sample sizes in further research. To examine changes in work routines directly, rather than indirectly via their assumed effects on well-being, would require using the DCM's subsidiary 'Personal Detractions' and 'Positive Events', or similar, coding frames. Lastly this study highlights the question of what specific organisational conditions help or hinder PDSA cycles and staff development activities in having an impact upon work routines in care homes, a barely researched topic. An expanded ethnographic research component that included, for example, direct observation of the DLC training and the dementia champions' meetings-in addition to the methods used in this study-would help answer questions around training and how its effects are achieved within care homes.

The DLC intervention, developed to strengthen the quality of the residential dementia care environment, combined a number of proven approaches including dementia training incorporating didactic and interactive elements [23, 58], continuous improvement processes [14-16], support through a community of practice [14], and recognition, coaching and reward of dementia care leadership and organisational culture change $[10,11]$. This evaluation which tested the underlying DLC assumptions (logic model) about the relationship between these intervention components and the intended outcomes offers evidence for areas where this dementia care practice innovation may be further refined.

\section{Abbreviations}

ADQ: Approaches to Dementia Questionnaire; DAS : Dementia Attitudes Scale; DCM : Dementia Care Mapping; DEMQOL: Dementia Quality of Life (questionnaire); DLC: Dementia Learning Community; GDS: Global

Deterioration Scale; NVQ : National Vocational Qualification; PDSA: Plan-DoStudy-Act (cycle); QCF : Qualification and Credit Framework (for training); QUALID: Quality of Life in Late-Stage Dementia (scale); SNCW : Satisfaction with Nursing Care and Work (assessment scale); TEP: Treatment Escalation Plan; WIB : Well/III Being (score)

\section{Acknowledgements}

This research was supported by the National Institute for Health Research (NIHR) Collaboration for Leadership in Applied Health Research and Care South West Peninsula. The views expressed are those of the authors, not necessarily those of the NHS, NIHR, South West Peninsula CLARHC or Department of Health. We are grateful to the two lead facilitators of the DLC project for their assistance with this study and to the reviewers for their suggestions, incorporated in the above paper, about logic model revisions. We also thank the care home residents, their relatives and staff who contributed to the study.

\section{Funding}

This research was funded by the Clinical Commissioning Group in which the Dementia Learning Community was piloted, and supported in kind by the National Institute for Health Research (NIHR) Collaboration for Leadership in Applied Health Research and Care South West Peninsula.

\section{Availability of data and materials}

The datasets analysed during the current study are available from the corresponding author on reasonable request, but in anonymised form only (as a condition of ethical approval) and provided that any costs of redacting individual, organisational and place names are met.

\section{Authors' contributions}

All three authors contributed to the study conception and design; to data acquisition, analysis and interpretation; and to drafting, critically revising and approving this manuscript. All authors read and approved the final manuscript.

\section{Ethics approval and consent to participate}

Plymouth University Research Ethics Committee gave ethical approval for this study on 21st February 2014 (Reference PU13/14-216), on condition that informants and care homes be pseudonymised in reports and publications about this study. We obtained informed consent from all participating care home managers and staff members; and from residents, whose willingness 
to participate was confirmed directly for those with mild to moderate dementia, or for those with severe dementia from a family member or other available proxy with designated authority to provide consent

\section{Consent for publication}

This consent is not applicable to this study.

\section{Competing interests}

The authors declare that they have no competing interests.

\section{Publisher's Note}

Springer Nature remains neutral with regard to jurisdictional claims in published maps and institutional affiliations.

\section{Author details \\ ${ }^{1}$ School of Government, Plymouth University, Drake Circus, Plymouth PL4 8AA, UK. ${ }^{2}$ Academic Partnership Lead for Dementia, Plymouth University Peninsula Schools of Medicine and Dentistry, Plymouth University, Drake Circus, Plymouth PL4 8AA, UK. ${ }^{3}$ Faculty of Social Sciences, Stirling University, Iris Murdoch Building, Stirling FK9 4LA, UK.}

\section{Received: 18 August 2016 Accepted: 25 January 2018} Published online: 05 February 2018

\section{References}

1. Boyle S. United Kingdom (England) health system review. Health Syst Transit. 2011;13:1-486.

2. Forsyth $\mathrm{G}$, Logan RFL. Medical technology and the needs of chronic disease: a review of some British studies on the organization of medical care services. J Chronic Dis. 1964;17:789-802.

3. Lyratzopoulos G, Havely D, Gemmell I, Good G. Factors influencing emergency medical readmission risk in a UK district general hospital: a prospective study. BMC Emerg Med. 2005:5:1.

4. Wilson T. Rising to the challenge: will the NHS support people with long term conditions? BMJ. 2005:330:657-61.

5. Alzheimer's Society. Low expectations: attitudes on choice, care and community for people with dementia in care homes. London: Alzheimer's Society; 2013

6. Department of Health. Developing high quality, effective, compassionate care: developing the right people with the right skills and values. Leeds: Health Education England; 2015.

7. Department of Health Commercial Directorate. Prime Minister's challenge on dementia 2020 [Internet]. London: Department of Health; 2015. Available from: https://www.gov.uk/government/publications/prime-ministerschallenge-on-dementia-2020

8. Franklin B. The future care workforce. London: ILC-UK; 2014

9. Evans S, Means R, Powell J. Making care homes part of the community? An evaluation of the Gloucestershire partnerships for older people project. Quality Ageing Older Adults. 2013;14:66-74.

10. Keating $\mathrm{M}$, Long J, Wright J. Leading culture change to improve dementia care. Nurs Times. 2013;109:16-8.

11. Doyle C. International perspectives on dementia education, training and knowledge transfer. Int Psychogeriatr. 2009;21:S1-2.

12. Banks P, Waugh A, Henderson J, Sharp B, Brown M, Oliver J, et al. Enriching the care of patients with dementia in acute settings? The dementia champions Programme in Scotland. Dementia. 2014;13:717-36.

13. Waugh A, Marland G, Henderson J, Robertson J, Wilson A. Improving the care of people with dementia in hospital. Nurs Stand. 2011;25:44-9.

14. Mayrhofer A, Goodman C, Holman C. Establishing a community of practice for dementia champions (innovative practice). Dementia. 2015;14:259-66.

15. Beer C, Lowry R, Horner B, Almeida OP, Scherer S, Lautenschlager NT, et al, Development and evaluation of an educational intervention for general practitioners and staff caring for people with dementia living in residential facilities. Int Psychogeriatr. 2011;23:221-9.

16. Mayrhofer A, Goodman C, Smeeton N. The role of dementia champion in dementia care: its aspirations, development and training needs (innovative practice). Dementia. 2016;15:1306-12

17. Bushe GR. Appreciative inquiry: theory and critique. The Routledge companion to organizational change; 2011. p. 87-103.

18. Argyris C. Single-loop and double-loop models in research on decision making. Adm Sci Q. 1976;21:363-75.
19. Cooksy LJ, Gill P, Kelly PA. The program logic model as an integrative framework for a multimethod evaluation. Eval Program Plann. 2001;24: 119-28.

20. NHS Institute for Innovation and Improvement. Plan, Do, Study, Act (PDSA) NHS Institute for Innovation and Improvement [Internet]. [cited 2016 May 23]. Available from: http://www.institute.nhs.uk/quality_and_service_ improvement_tools/quality_and_service_improvement_tools/plan_do_ study_act.html

21. Taylor N, Clay-Williams R, Hogden E, Braithwaite J, Groene O. High performing hospitals: a qualitative systematic review of associated factors and practical strategies for improvement. BMC Health Serv Res. 2015;15:244.

22. Taylor MJ, McNicholas C, Nicolay C, Darzi A, Bell D, Reed JE. Systematic review of the application of the plan-do-study-act method to improve quality in healthcare. BMJ Qual Saf. 2013;bmjqs-2013-001862.

23. Featherstone $K$, James IA, Powell I, Milne D, Maddison C. A controlled evaluation of a training course for staff who work with people with. Dementia. 2004:3:181-94.

24. Woods B. Why is training not enough to make a diference in dementia care [Internet]. Bangor; ND. Available from: http://www.google.co.uk/url?sa= $t \& r c t=j \& q=\& e s r c=s \&$ source $=$ web\&cd $=1 \&$ ved $=0 C C g Q F j A A a h U K E w i y 9 o$ Khc3GAhXmin|KHXJEBao\&url=http\%3A\%2F\%2Fdementia. ie\%2Fimages\%2Fuploads\%2Fsite-images\%2FBobWoods.pdf\&ei=OGdVbKaBuadygPyiJXQCg\&usg=AFQjCNFT3_ PMwzh32civ7mepmMOoQa5BNA\&bvm =bv.96952980,d.bGQ.

25. Pike L. Not Just Ticking the Box: An Investigation into Safeguarding Adults Training Transfer in Cornwall, UK [PhD thesis]. [Plymouth University]: Plymouth; 2013

26. Jeon $Y-H$, Luscombe G, Chenoweth L, Stein-Parbury J, Brodaty H, King M, et al. Staff outcomes from the caring for aged dementia care REsident study (CADRES): a cluster randomised trial. Int J Nurs Stud. 2012:49:508-18.

27. Chenoweth L, King MT, Jeon $Y-H$, Brodaty H, Stein-Parbury J, Norman R, et al. Caring for aged dementia care resident study (CADRES) of personcentred care, dementia-care mapping, and usual care in dementia: a clusterrandomised trial. Lancet Neurol. 2009:8:317-25.

28. Ballard C, Aarsland D. Person-centred care and care mapping in dementia. Lancet Neurol. 2009;8:302-3.

29. Macdonald AJD, Woods RT. Attitudes to dementia and dementia care held by nursing staff in U.K. care homes: what difference do they make? Int Psychogeriatr. 2005;17:383-91.

30. Berwick DM. A primer on leading the improvement of systems. BMJ. 1996; 312:619-22.

31. Volicer L, Mills PD, Hurley AC, Warden V. Home care for patients with dementia. Federal Practitioner. 2004;

32. Uyanwune CO. Non-Pharmacological Management of The Behavioral And Psychological Symptoms of Dementia [DNP thesis]. Amherst: University of Massachusetts; 2014

33. Caminal J, Starfield B, Sanchez E, Casanova C, Morales M. The role of primary care in preventing ambulatory care sensitive conditions. Eur J Pub Health. 2004:14:246-51.

34. Baier RR, Gifford DR, Patry G, Banks SM, Rochon T, DeSilva D, et al. Ameliorating pain in nursing homes: a collaborative quality-improvement project. J Am Geriatr Soc. 2004;52:1988-95.

35. Milward HB, Provan KG, Fish A, Isett KR, Huang K. Governance and collaboration: an evolutionary study of two mental health networks. J Public Adm Res Theory. 2009;20:1125-41.

36. Pawson R. Evidence-based policy. London: Sage; 2008.

37. Pinzur MS, Gurza E, Kristopaitis T, Monson R, Wall MJ, Porter A, et al. Hospitalist-orthopedic co-Management of High-Risk Patients Undergoing Lower Extremity Reconstruction Surgery. Orthopedics. 2009;32:495-501.

38. Porter B, Hjern D. Implementation structures: new unit of administrative analysis. Organ Stud. 1981;2:211-27.

39. Saunders RP, Evans MH, Joshi P. Developing a process-evaluation plan for assessing health promotion programme implementation: a how-to guide. Health Promot Pract. 2005:6:134-47.

40. Fetterman DM. Ethnography. London: Sage; 1998.

41. Lofland J, Lofland LH. Analyzing social settings. A guide to qualitative observation and analysis. 3rd ed. London: Wadsworth; 1995.

42. Silverman D. Doing qualitative research - a practical handbook. London: Sage; 2000.

43. O'Connor ML, McFadden SH. Development and psychometric validation of the dementia attitudes scale. Int J Alzheimers Dis. 2010;2010:e454218. 
44. Lintern T, Woods B. Approaches to dementia questionnaire. Bangor: University of Wales; 1996

45. Brodaty $H$, Draper B, Low L. Nursing home staff attitudes towards residents with dementia: strain and satisfaction with work. J Adv Nurs. 2003;44:583-90.

46. Bradford Dementia Group. DCM 8 user's manual: the DCM method. 8th ed. Bradford: University of Bradford; 2005.

47. Brooker D. Dementia care mapping: a review of the literature. The Gerontologist. 2005;45:11-8.

48. Fossey J, Lee L, Ballard C. Dementia care mapping as a research tool for measuring quality of life in care setting: psychometric properties. Int J Geriatric Psychiatry. 2002;17:1064-70.

49. Reisberg B, Ferris S, de Leon MJ, Crook T. The global deterioration scale for assessment of primary degenerative dementia. Am J Psychiatry. 1982:139:1136-9.

50. Smith SC, Lamping DL, Banerjee S, Harwood RH, Foley B, Smith P, et al. Development of a new measure of health-related quality of life for people with dementia: DEMQOL. Psychol Med. 2007;37:737-46.

51. Weiner M, Martin-Cook K, Svetlik D, Saine K, Foster B, Fontaine C. The quality of life in late-stage dementia (QUALID) scale. J Am Med Dir Assoc. 2000;:1:114-6,

52. Hurt CS, Banerjee S, Tunnard C, Whitehead DL, Tsolaki M, Mecocci P, et al. Insight, cognition and quality of life in Alzheimer's disease. J Neurol Neurosurg Psychiatry. 2010;81:331-6.

53. Benhabib H, Lanctot KL, Eryavec GM, Li A, Herrmann N. Responsiveness of the QUALID to improved neuropsychiatric symptoms in patients with Alzheimer's disease. Can Geriatr J. 2013;16:180-5.

54. Sherratt C, Soteriou T, Evans S. Ethical issues in social research involving people with. Dementia. 2007;6:463-79.

55. Auer S, Reisberg B. The GDS/FAST staging system. Int Psychogeriatr. 1997;9:167-71.

56. Coleman MT, Looney S, O'Brien J, Ziegler C, Pastorino CA, Turner C. The Eden Alternative. Findings after 1 year of implementation. J Gerontol Ser A Biol Med Sci. 2002;57:M422-7.

57. Fleming R, FitzGerald D. Large-scale training in the essentials of dementia care in Australia: dementia care skills for aged care workers project. Int Psychogeriatr. 2009;21:S53-7.

58. Landreville $P$, Dicaire $L$, Verreault $R$, Lévesque $L$. A training program for managing agitation of residents in long-term care facilities: description and preliminary findings. J Gerontol Nurs. 2005;31:34-42.

\section{Submit your next manuscript to BioMed Central and we will help you at every step:}

- We accept pre-submission inquiries

- Our selector tool helps you to find the most relevant journal

- We provide round the clock customer support

- Convenient online submission

- Thorough peer review

- Inclusion in PubMed and all major indexing services

- Maximum visibility for your research

Submit your manuscript at www.biomedcentral.com/submit
Biomed Central 